\title{
Nivolumab for Advanced Hepatocellular Carcinoma with Multiple Lung Metastases after Sorafenib Failure
}

\author{
Jaewoong Kim', Jin Won Chang', Jun Yong Park ${ }^{1,2,3}$ \\ 'Department of Internal Medicine, ${ }^{2}$ Institute of Gastroenterology, Yonsei University College of Medicine, Seoul; ${ }^{3}$ Yonsei Liver Center, \\ Yonsei University Health System, Seoul, Korea
}

Received Aug. 11, 2019

Revised Feb. 6, 2020

Accepted Feb. 7, 2020
Over the past decade, standard first-line systemic treatment of advanced hepatocellular carcinoma (HCC) has been based on sorafenib, a multi-kinase inhibitor. Regorafenib, another tyrosine kinase inhibitor, is the only second-line therapy that has been globally approved after progression under sorafenib treatment. Recently, immunotherapeutic agents have emerged as promising treatment options in many different malignancies, including advanced HCC. Nivolumab is the first immunotherapy approved by the Food and Drug Administration for use in $\mathrm{HCC}$ patients with advanced-stage second-line after sorafenib failure. In this report, a case of advanced HCC with multiple lung metastases in which a complete response and maintained progression-free status was achieved with nivolumab, following the failure of transarterial chemoembolization and sorafenib is presented. We hope this report may help expand the clinical application of second-line treatment. (J Liver Cancer 2020;20:72-77)

Keywords: Hepatocellular carcinoma; Lung metastasis; Sorafenib failure; Immunotherapy; Nivolumab

\section{INTRODUCTION}

Hepatocellular carcinoma (HCC) is the most common primary malignant tumor in the liver and the second most frequent cause of cancer-related death globally. ${ }^{1}$ In Korea, HCC has the highest mortality rate in the working-age population, and the greatest economic burden of cancer. ${ }^{2}$ Surgical treatment or local ablation is the only curative therapy for localized disease. Therapeutic options for advanced disease are even more limited. ${ }^{1-3}$ Sorafenib, the first molecularly targeted systemic agent for advanced liver cancer, has remained the primary treatment option since $2007 .{ }^{4-6}$ Another multi-ki-

\footnotetext{
Corresponding author : Jun Yong Park

Department of Internal Medicine, Yonsei University College of Medicine, 50-1 Yonsei-ro, Seodaemun-gu, Seoul 03722, Korea

Tel. +82-2-2228-1988, Fax. +82-2-393-6884

E-mail;drpjy@yuhs.ac.kr

http://orcid.org/0000-0001-6324-2224
}

nase inhibitor, regorafenib, was approved for the treatment of HCC patients who have been previously treated with sorafenib but showed limited survival benefits. ${ }^{7}$ A more effective systemic treatment option for advanced HCC is strongly warranted. In recent years, new immune-modulatory agents have been introduced for the oncological treatment of various solid malignancies. Immune checkpoint proteins, inhibiting the interaction between programmed death receptor 1 (PD-1) and its ligand have shown substantial survival benefits in many different malignancies. ${ }^{8-10}$ Nivolumab, as a novel immune checkpoint inhibitor, is increasingly used in patients with cancer, in particular for those with melanoma. Nivolumab is currently being evaluated in relation to a number of other cancers such as metastatic non-small cell lung cancer following treatment failure of platinum-based chemotherapy, advanced renal cell cancer and squamous cell carci- 
noma of the head and neck. ${ }^{11-13}$ This treatment can lead to a powerful multi-target immune response that can induce persistent oncological remission in some patients. A phase I/II CheckMate-040 study investigated nivolumab treatment in patients with advanced HCC. In the dose-expansion phase of this study, patients received nivolumab $3 \mathrm{mg} / \mathrm{kg}$, and the objective response rate was $20 \%$, with a median duration of response of 9.9 months and a 9-months overall survival rate of $74 \% .{ }^{14}$ The nivolumab safety profile was consistent with that observed in other tumor types, and no new safety signals were observed. Here, we report a case of advanced HCC with multiple lung metastases that responded dramatically to nivolumab treatment after transarterial chemoembolization (TACE) and sorafenib failure, which are the current standard therapies for the advanced/metastatic stage.

\section{CASE REPORT}

A 57-year-old male with chronic hepatitis B was referred to our hospital from a local clinic due to elevated serum $\alpha$ fetoprotein (AFP) and suspicious HCC on computed tomography (CT). On magnetic resonance imaging, multiple masses in both hemiliver were well enhanced on the arterial phase and rapidly washed out on the portal phase, consistent

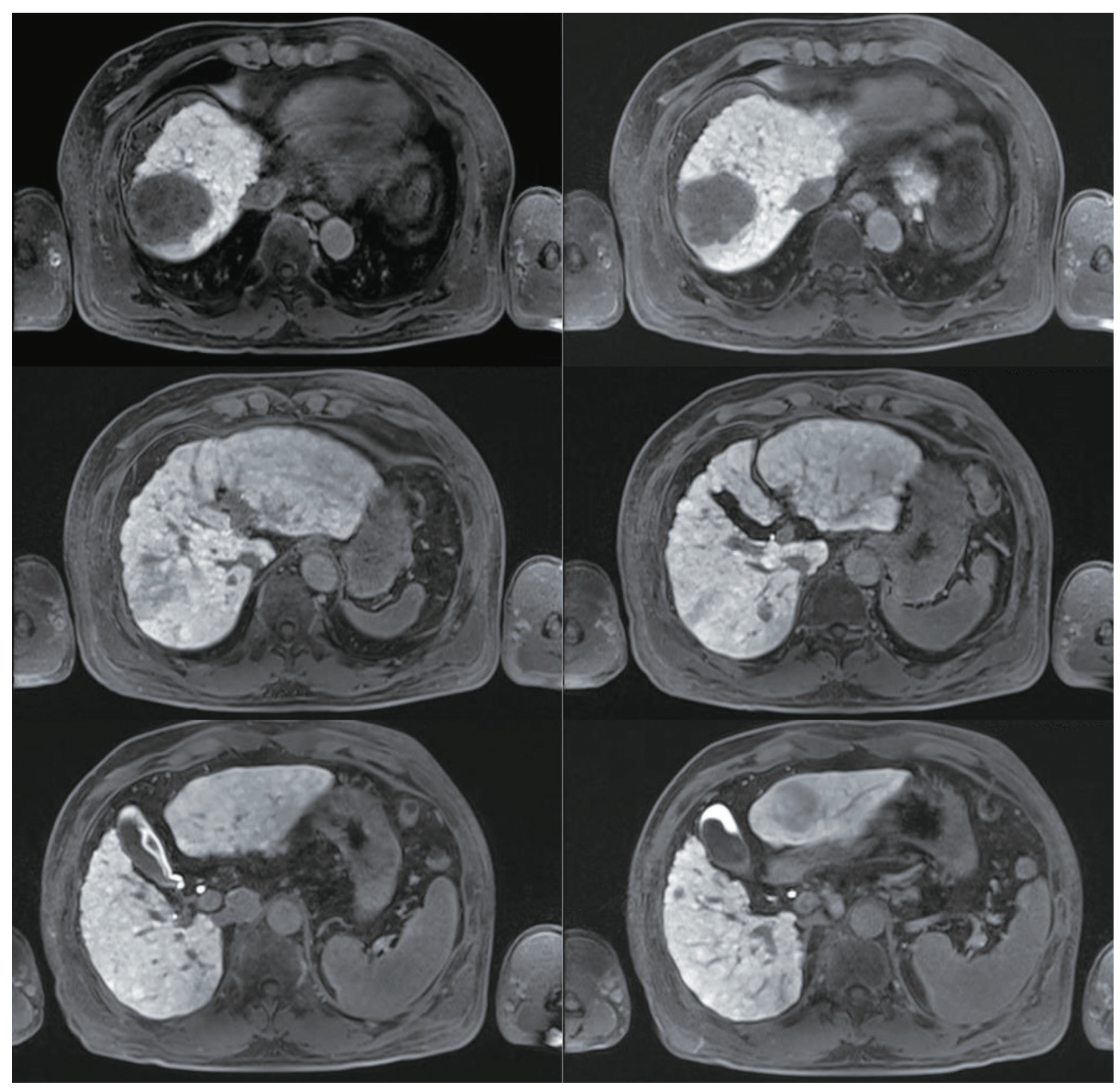

Figure 1. Magnetic resonance imaging findings. Multiple liver masses are seen; $5.8 \mathrm{~cm}$ in the dome, $4.1 \mathrm{~cm}$ in the $S 3$, and smaller nodules in the $\mathrm{S} 1,2.4 \mathrm{~cm} ; \mathrm{S} 7,0.7 \mathrm{~cm} \& 1.3 \mathrm{~cm}$; and $\mathrm{S} 5,0.9 \mathrm{~cm}$. 
with HCC (Fig. 1). There was no evidence of extrahepatic metastasis. The serum AFP level was 1,109 ng/mL, and the protein level induced by vitamin $\mathrm{K}$ absence-II (PIVKA-II) was $5,808 \mathrm{mAU} / \mathrm{mL}$. The initial liver function test was normal, with alanine aminotransferase $18 \mathrm{IU} / \mathrm{L}$, total bilirubin $1.1 \mathrm{mg} / \mathrm{dL}$, albumin $4.1 \mathrm{~g} / \mathrm{dL}$, prothrombin time international normalized ratio of 1.00 and HBV DNA $120 \mathrm{IU} / \mathrm{mL}$. The patient had no portal hypertension, encephalopathy or ascites, and performance status was good, with an Eastern Cooperative Oncology Group score of zero. Modified Union for International Cancer Control was stage III, T3NOM0, and the Barcelona Clinic Liver Cancer (BCLC) stage was considered to be intermediate (BCLC B). Therefore, TACE was performed three times as a loco-regional treatment while

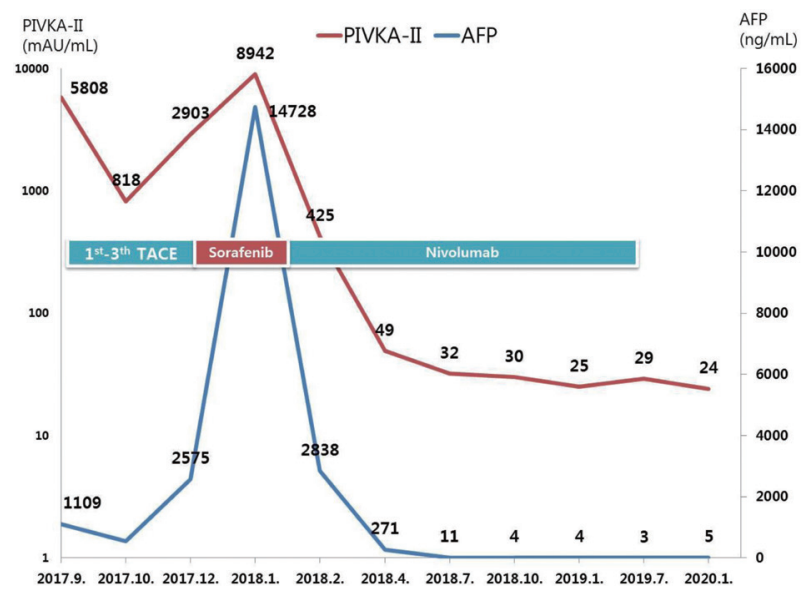

Figure 2. The clinical course of serum AFP and PIVKA-II levels and the timing of the therapeutic methods. AFP, $\alpha$-fetoprotein; PIVKA-II, protein induced by vitamin $\mathrm{K}$ absence-II; TACE, transarterial chemoembolization. confirming the treatment response. However, imaging tests performed to assess the TACE response detected hematogenous lung metastasis and tumor thrombus in the right hepatic vein (HV), and AFP and PIVKA-II levels elevated to $2,575 \mathrm{ng} / \mathrm{mL}$ and 2,903 mAU/ml (Fig. 2). For systemic treatment, the patient started $400 \mathrm{mg}$ of sorafenib orally, twicedaily. Sorafenib was tolerable without any severe side effects and was maintained for 6 weeks without dose reduction. However, the multiple lung metastases continued to increase on chest radiograph and chest CT with increased AFP and PIVKA-II (Fig. 3A, 4A). Also, tumor thrombus in the right HV slightly increased (Fig. 5A). After sorafenib failure as the first systemic therapy, a bi-weekly treatment with intravenous nivolumab (Obdivo $\left.{ }^{\mathrm{TM}}\right) 225 \mathrm{mg}(3 \mathrm{mg} / \mathrm{kg}$ ) was given. After 2 cycles ( 4 weeks) of nivolumab therapy, AFP and PIVKA-II levels decreased markedly with a significant decrease in the multiple lung metastases and retracted right $\mathrm{HV}$ thrombosis (Fig. 2-5). There was no drug toxicity. Six months after beginning nivolumab therapy, the AFP and PIVKA-II levels were normalized, and the multiple lung metastases disappeared. The tumor response to nivolumab was assessed as a complete response (CR) by the modified response evaluation criteria of the solid tumors criteria. The patient underwent 24 cycles of nivolumab therapy (48 weeks after starting nivolumab) to remove any tumor cell remnants. The patient has no signs of disease recurrence 12 months after stopping the nivolumab treatment (Fig. 2, 3).

This case report is limited to one case and did not cause harm to the study subject. No sensitive information was collected or recorded from the subject. For this reason, institu-
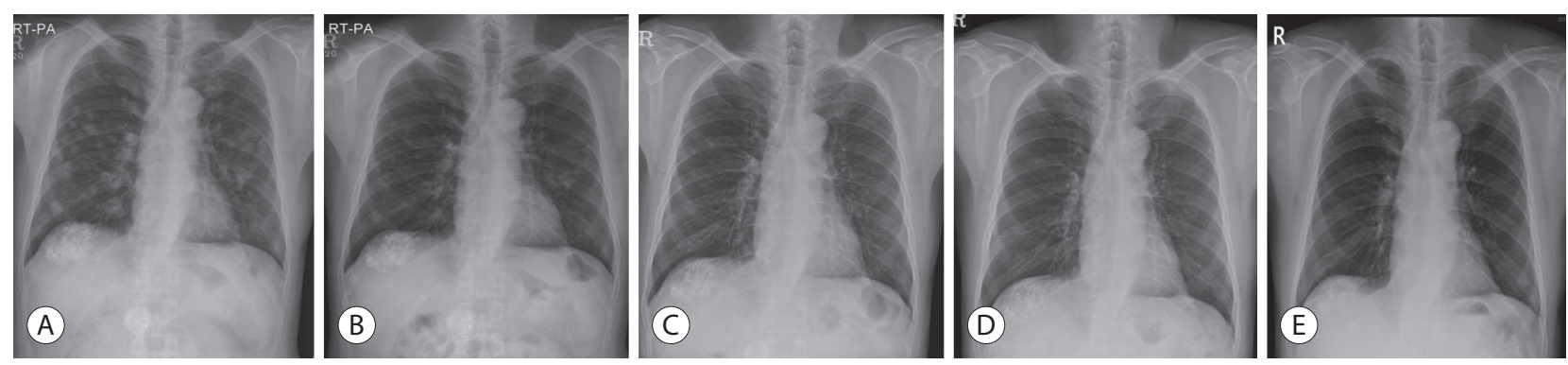

Figure 3. Chest radiographs. Follow-up after complete remission achieved, showing no recurrence of the tumor. (A) Before nivolumab therapy. (B) After 4 weeks of nivolumab therapy. (C) After 24 weeks of nivolumab therapy. (D) After 48 weeks of nivolumab therapy. (E) Forty-eight weeks after the end of treatment. 

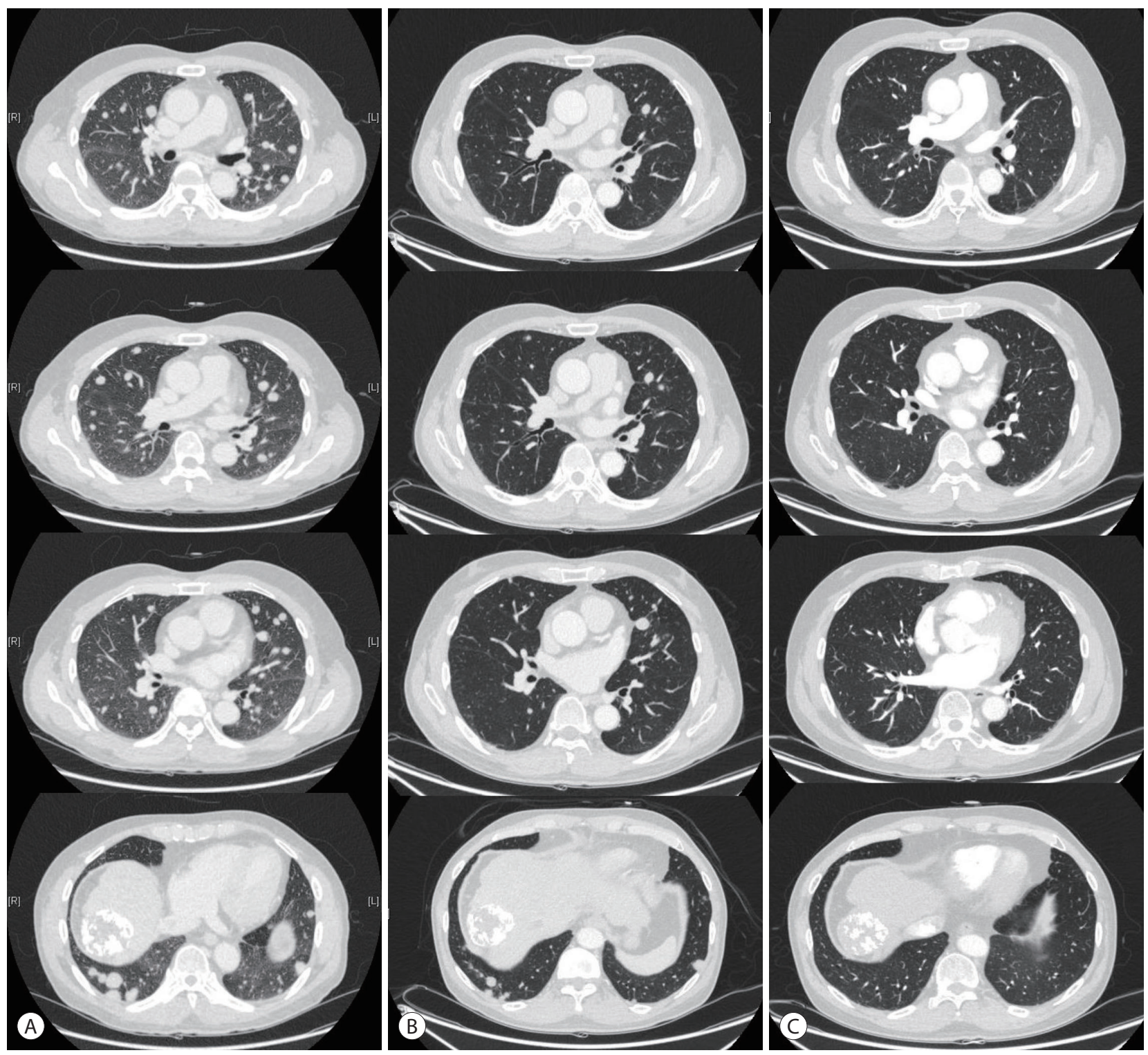

Figure 4. Chest contrast-enhanced computed tomography. (A) Before nivolumab therapy. (B) After 4 weeks of nivolumab therapy. (C) After 12 weeks of nivolumab therapy.
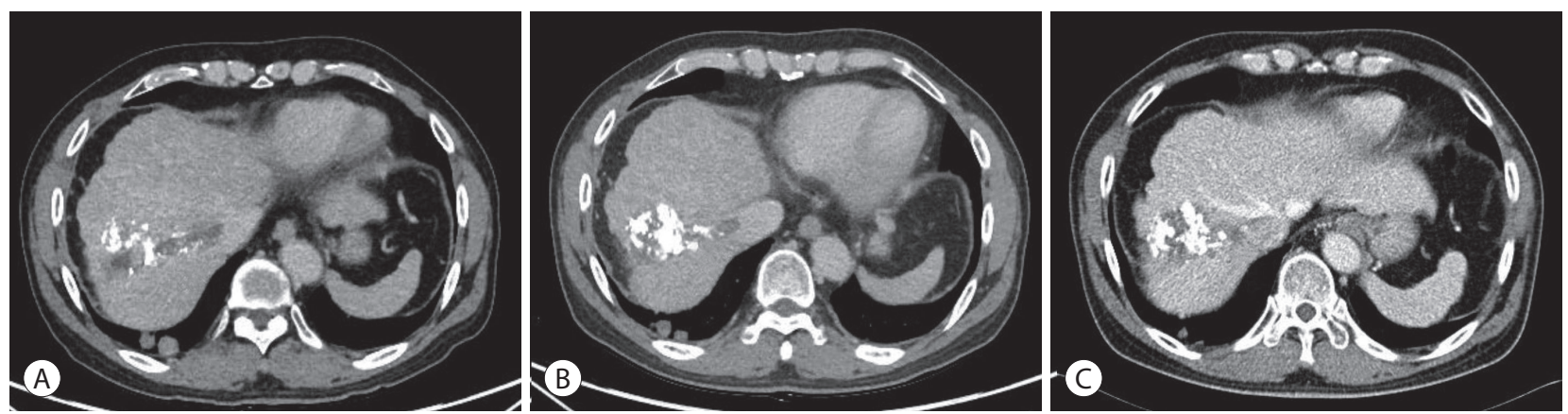

Figure 5. Abdomen contrast-enhanced computed tomography. (A) Before nivolumab therapy. (B) After 4 weeks of nivolumab therapy. (C) After 12 weeks of nivolumab therapy. 
tional review board (IRB) approval was waived.

\section{DISCUSSION}

HCC is the most common form of liver cancer. However, treatment options are limited, with the standard options only adding a three-month survival benefit over a placebo. In the systemic treatment of advanced HCC, sorafenib has been the first choice of treatment since 2007..$^{4-6}$ Many other agents have been developed after sorafenib, such as sunitinib, linifanib, brivanib. However, these agents displayed no improvement in the survival rate. In recent years, immunotherapy has been highlighted in various solid malignancies, and immune-oncology has emerged in advanced HCC. ${ }^{15-17}$ Beyond the limits of standard therapies, immunotherapy strategies for advanced HCC are emerging with a number of innovative drugs. Nivolumab, the first PD-1 agent which blocks inhibitory $\mathrm{T}$ cell checkpoints, have shown great promise in clinical trials involving melanoma, non-small cell lung cancer, and renal cell cancer. ${ }^{11-13}$ The CheckMate-040 study, which was a multi-cohort phase I/II trial that investigated nivolumab demonstrated that it is a promising treatment choice in advanced HCC. ${ }^{14}$ The study proved the efficacy and objective response rate in patients with intermediate-advanced HCC and Child-Pugh stage A. Additionally, nivolumab was well tolerated, with only $6 \%$ of patients presenting serious treatment-related adverse events. Therefore, the Food and Drug Administration (FDA) granted nivolum$\mathrm{ab}$ conditional approval as a second-line agent for advanced HCC in September 2017.

Following the favorable results of recent studies, nivolum$\mathrm{ab}$ was selected as an option for the patient discussed in this report. As nivolumab is not covered by the National Health Insurance System of Korea, its use can be expensive. However, after a discussion, our patient was satisfied with the favorable results and willing to try the treatment. In our case, nivolumab exceeded our expectations, not only achieving CR of the tumor but also showing greater progression-free survival (PFS) and duration of response after the treatment was discontinued. During nivolumab treatment, our patient had excellent results without any treatment-related adverse ef- fects. We stopped nivolumab therapy after 24 cycles (48 weeks) based on the performance of the patient and PFS. Treatment costs were important in determining and maintaining treatment. As in this case, there is still no consensus on how long chemotherapy, including immunotherapy, should be kept when CR enters.

Recently, in the CheckMate-049 study, a phase-3 randomized study of nivolumab monotherapy was compared to sorafenib in the first-line setting. In this study, nivolumab did not display a statistically significant improvement in overall survival, which was the primary endpoint. ${ }^{18}$ However, nivolumab might provide favorable efficacy with a good safety profile in the context of the available targeted therapies. Like other malignancies, finding biomarkers that can predict treatment response to immunotherapy, including nivolum$\mathrm{ab}$, is of paramount importance in advanced HCC. ${ }^{19}$

In this report, we demonstrated CR using nivolumab for metastatic HCC after sorafenib failure. We hope this report will help expand the clinical application of immunotherapy and may be an alternative treatment option for treating advanced/metastatic HCC.

\section{Conflicts of Interest}

The authors have no conflicts to declare.

\section{REFERENCES}

1. Villanueva A. Hepatocellular carcinoma. N Engl J Med 2019;380:1450-1462.

2. Korean Liver Cancer Association; National Cancer Center. 2018 Korean Liver Cancer Association-National Cancer Center Korea Practice Guidelines for the Management of Hepatocellular Carcinoma. Gut Liver 2019;13:227-299.

3. Lee JS, Cho IR, Lee HW, Jeon MY, Lim TS, Baatarkhuu O, et al. Conditional survival estimates improve over time for patients with hepatocellular carcinoma: An Analysis for Nationwide Korea Cancer Registry Database. Cancer Res Treat 2019;51:1347-1356.

4. Llovet JM, Ricci S, Mazzaferro V, Hilgard P, Gane E, Blanc JF, et al. Sorafenib in advanced hepatocellular carcinoma. N Engl J Med 2008;359:378-390,

5. Cheng AL, Kang YK, Chen Z, Tsao CJ, Qin S, Kim IS, et al. Efficacy and safety of sorafenib in patients in the Asia-Pacific region with advanced hepatocellular carcinoma: a phase III randomised, dou- 
ble-blind, placebo-controlled trial. Lancet Oncol 2009;10:25-34.

6. Cheng AL, Guan Z, Chen Z, Tsao CJ, Qin S, Kim JS, et al. Efficacy and safety of sorafenib in patients with advanced hepatocellular carcinoma according to baseline status: subset analyses of the phase III Sorafenib Asia-Pacific trial. Eur J Cancer 2012;48:14521465.

7. Bruix J, Qin S, Merle P, Granito A, Huang YH, Bodoky G, et al. Regorafenib for patients with hepatocellular carcinoma who progressed on sorafenib treatment (RESORCE): a randomised, doubleblind, placebo-controlled, phase 3 trial. Lancet 2017;389:56-66.

8. Pardoll DM. The blockade of immune checkpoints in cancer immunotherapy. Nat Rev Cancer 2012;12:252-264.

9. Emens LA, Ascierto PA, Darcy PK, Demaria S, Eggermont AMM, Redmond WL, et al. Cancer immunotherapy: opportunities and challenges in the rapidly evolving clinical landscape. Eur J Cancer 2017:81:116-129.

10. Whiteside TL, Demaria S, Rodriguez-Ruiz ME, Zarour HM, Melero I. Emerging opportunities and challenges in cancer immunotherapy. Clin Cancer Res 2016;22:1845-1855.

11. Kang YK, Boku N, Satoh T, Ryu MH, Chao Y, Kato K, et al. Nivolumab in patients with advanced gastric or gastro-oesophageal junction cancer refractory to, or intolerant of, at least two previous chemotherapy regimens (ONO-4538-12, ATTRACTION-2): a randomised, double-blind, placebo-controlled, phase 3 trial. Lancet 2017;390:2461-2471.

12. Larkin J, Chiarion-Sileni V, Gonzalez R, Grob JJ, Cowey CL, Lao $C D$, et al. Combined nivolumab and ipilimumab or monotherapy in untreated melanoma. N Engl J Med 2015;373:23-34.

13. Harrington KJ, Ferris RL, Blumenschein $G \mathrm{Jr}$, Colevas $A D$, Fayette
J, Licitra L, et al. Nivolumab versus standard, single-agent therapy of investigator's choice in recurrent or metastatic squamous cell carcinoma of the head and neck (CheckMate 141): health-related quality-of-life results from a randomised, phase 3 trial. Lancet Oncol 2017;18:1104-1115.

14. El-Khoueiry AB, Sangro B, Yau T, Crocenzi TS, Kudo M, Hsu C, et al. Nivolumab in patients with advanced hepatocellular carcinoma (CheckMate 040): an open-label, non-comparative, phase 1/2 dose escalation and expansion trial. Lancet 2017;389:2492-2502.

15. Chon YE, Park H, Hyun HK, Ha Y, Kim MN, Kim BK, et al. Development of a new Nomogram including neutrophil-to-lymphocyte ratio to predict survival in patients with hepatocellular carcinoma undergoing transarterial chemoembolization. Cancers (Basel) 2019;11:E509.

16. Cho Y, Han J, Kim W. Recent advances and future directions in immunotherapeutics for hepatocellular carcinoma. J Liver Cancer 2019;19:1-11.

17. Lee HW, Cho KJ, Park JY. Current status and future direction of immunotherapy in hepatocellular carcinoma: what do the data suggest? Immune Netw 2020;20:e11.

18. Yau T, Park J, Finn R, Cheng A, Mathurin P, Edeline J, et al. CheckMate 459: A randomized, multi-center phase III study of nivolumab (NIVO) vs sorafenib (SOR) as first-line (1L) treatment in patients (pts) with advanced hepatocellular carcinoma (aHCC). Ann Oncol 2019;30:v874-v875.

19. Lee HW, Cho KJ, Shin SY, Kim HY, Lee EJ, Kim BK, et al. Serum PD-1 levels change with immunotherapy response but do not predict prognosis in patients with hepatocellular carcinoma. J Liver Cancer 2019;19:108-116. 